\title{
Nanoscale
}

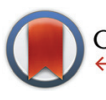

CrossMark \& click for updates

Cite this: Nanoscale, 2015, 7, 1857

Received 9th October 2014

Accepted 9th December 2014

DOI: $10.1039 / c 4 n r 05934 d$

www.rsc.org/nanoscale

\section{Stamping colloidal photonic crystals: a facile way towards complex pixel colour patterns for sensing and displays $\uparrow$}

\author{
Tao Ding, ${ }^{\text {a,b }}$ Stoyan K. Smoukov ${ }^{\mathrm{b}}$ and Jeremy J. Baumberg ${ }^{{ }^{a}}$
}

Patterning of colloidal photonic crystals (CPCS) has been strongly investigated in recent years for sensing and image displays. Rather than using traditional template-directed approaches, here microimprint lithography along with convective self-assembly is applied to generate complex CPC patterns that can be adjusted to show single- or dual-colour patterns or composite CPC patterns possessing two different colours. These composite CPC patterns show different wettability with water because of the surface chemistry of the polymers and silica used. This dramatically transforms the structural colours upon liquid infiltration. By mixing different ethanol concentrations with water, the infiltration efficiency can be further improved and easily read out from changes in reflection intensity and spectral peak shifts. Integrating these nano-architectures into devices can thus yield function as image displays and as sensors for solvents.

\section{Introduction}

A colloidal photonic crystal (CPC) is a periodically arranged array of submicron particles that selectively inhibits the transmission of light, or traps certain optical wavelengths. These CPCs hold great promise for next-generation optical computation and communication components, because of their scalable, cost-effective and straightforward fabrication. ${ }^{1-5}$ Because of this, patterning of CPCs has also been intensively studied over the last decade. ${ }^{6}$ However, unintentional defects and cracks along with incomplete bandgaps still leave CPCs far from being able to target such photonics devices. ${ }^{7-9}$ On the other hand, colour pattern generation that can respond to environmental changes or stimuli has been intensively investigated in the last few years for sensing and image displays. ${ }^{10-15}$ This integrated colour patterning system has unique advantages over competitive organic colour systems (as CPCs are non-toxic, environmentally friendly and non-photo-bleaching) and can be actively tuned with external fields for displays or combined with different materials for sensing applications.

\footnotetext{
${ }^{a}$ Nanophotonics Centre, Cavendish Laboratory, University of Cambridge, CB3 OHE, UK.E-mail:dt413@cam.ac.uk, jjb12@cam.ac.uk; Tel: +44 (0)1223 760945

${ }^{b}$ Department of Materials Science and Metallurgy, 27 Charles Babbage Road; University of Cambridge, CB3 OFS, UK. Fax: +44 (0)1223 762088; Tel: $+44(0) 1223334435$

$\dagger$ Electronic supplementary information (ESI) available: SEM images of the stamps, optical image, reflection spectra, and table of peak wavelength and intensity. See DOI: 10.1039/c4nr05934d
}

The techniques that have been developed for patterning CPCs involve lithography, ${ }^{16-18}$ template-directed selfassembly ${ }^{19-28}$ and other less conventional approaches like capillary assisted deposition ${ }^{29}$ and injet printing. ${ }^{30-32}$ However, the processes are either complicated and time consuming or expensive. For example, lithography approaches require sophisticated mask alignment for UV exposures and the procedure is quite complicated. ${ }^{16}$ The templating approaches require fabrication of pre-patterned chemical or physical templates which can only be used once. The unconventional approaches, ${ }^{29,33}$ though scalable, are only applicable to certain types of patterns with simple arrangements, and are thus not very versatile or generic. Therefore facile fabrication of large-scale patterned CPCs still remains a great challenge but is strongly desirable.

Nano- and micro-imprinting techniques are prominent for large-scale surface patterning of polymer films but seldom have been applied for patterning of CPCs. ${ }^{34}$ The group of Yang et al. attempted to apply microcontact printing ${ }^{35,36}$ to pattern colloidal crystal films but this resulted in only surface patterned morphologies with no distinctive structural colours. Key to the success of applying microimprinting techniques to pattern CPCs is the control and modification of the mechanical stability of the CPC films, which is strongly dependent on the imprinting temperature and pressure. Here, we fabricate complex pixel patterns of CPCs based on microimprint lithography techniques which provide a highly facile and costeffective way for large scale patterning of CPCs. Two scenarios are discussed here. If the CPCs made of poly(styrene-methyl 
methacrylate-acrylic acid) (Poly(St-MMA-AA)) are annealed before imprinting, the whole film maintains its integrity but cannot easily be lifted-off from the stamps. In this case the structural colours of the printed regions are blue-shifted because of the decreased lattice spacing along the film normal due to compression. Therefore a second approach was also used, in which CPCs with dual colours are generated. Without any pre-annealing, the locally imprinted regions can be entirely lifted-off by the stamps, thus generating negative patterns on the substrate. Depositing another layer of silica CPCs in-between Poly(St-MMA-AA) CPC patterns leads to dual colour patterns. Such pixel colour features of these CPC patterns can potentially be applied in image display applications. Because of the hydrophobicity difference between silica and Poly(StMMA-AA), the optical response of the two patterns is also quite different in different solvents. Therefore, these films can be used as sensors with direct colour readout. The different response of the patterns to solvents can also be applied in anti-counterfeiting applications.

\section{Results and discussion}

The fabrication process is schematically illustrated in Scheme 1. First, CPC film layers are formed via convective selfassembly from colloidal Poly(St-MMA-AA) sphere solutions. Before microimprinting, the CPC films are annealed at $90{ }^{\circ} \mathrm{C}$ for $30 \mathrm{~min}$ to enhance their mechanical stability by sintering the contacted spheres. During the imprinting process, an epoxy resin stamp with hexagonally arranged pillars was used (ESI, Fig. S1a †े), and the imprinted regions are compressed and the Poly(St-MMA-AA) nanospheres partially melt and deform, since the temperature used is slightly above the glass transition. Therefore, the structural colour of these imprinted regions (labelled Region II) are blue-shifted while the nonimprinted regions (Region I) remain the same. As a result, green and yellow dual patterns are formed across the films as shown in the inset of Fig. 1a. The cracks form during the original drying process of the CPC films, and currently cannot be fully avoided, although many other groups are separately working on this aspect. ${ }^{37-39}$ Fig. 1a shows the corresponding SEM image of the patterns in the CPC films. At the boundary of the patterns, the different surface morphology is clearly seen - this edge between imprinted and non-imprinted regions is shown in Fig. 1b, which shows an expanded view of the white box in Fig. 1a. Cross-sectional views of these patterns further reveal the change of nano-architecture in the film

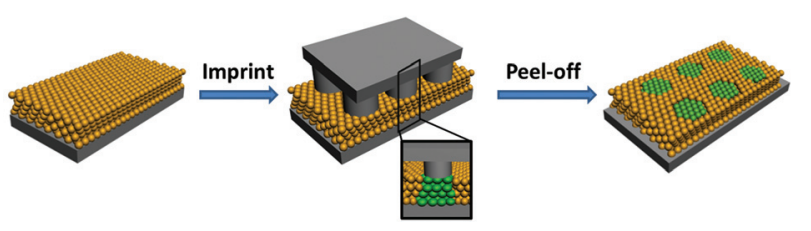

Scheme 1 The fabrication procedure of dual patterned CPCs via microimprint lithography.
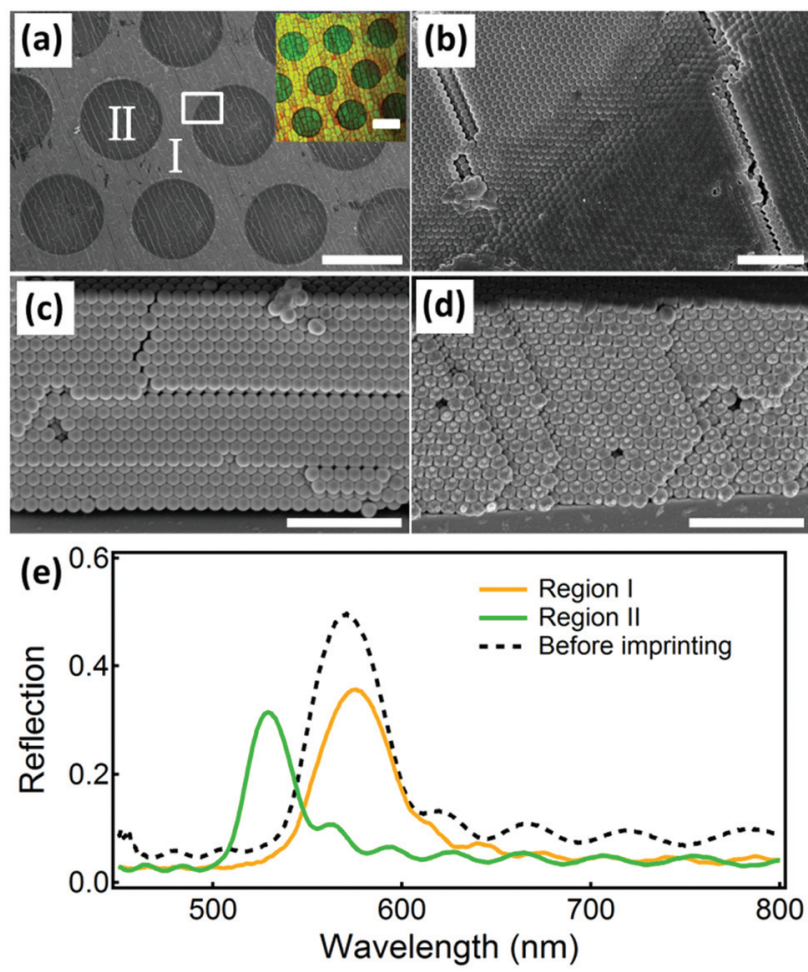

Fig. 1 SEM images of the imprinted CPC patterns made of Poly(StMMA-AA), $90{ }^{\circ} \mathrm{C}, 30$ bar, $60 \mathrm{~s}$. (a) Top-view of the micropatterns. Inset is the optical image, scale bar is $100 \mu \mathrm{m}$. (b) Magnified view of the boundary of the micropatterns inside white box in 1a. (c, d) Cross-sectional views of the non-imprinted and imprinted patterns. All scale bars in SEMs are $2 \mu \mathrm{m}$. (e) Reflection spectra of the non-imprinted (Region I) and printed regions (Region II). Dashed line is reflection of Poly(StMMA-AA) CPCs before imprinting.

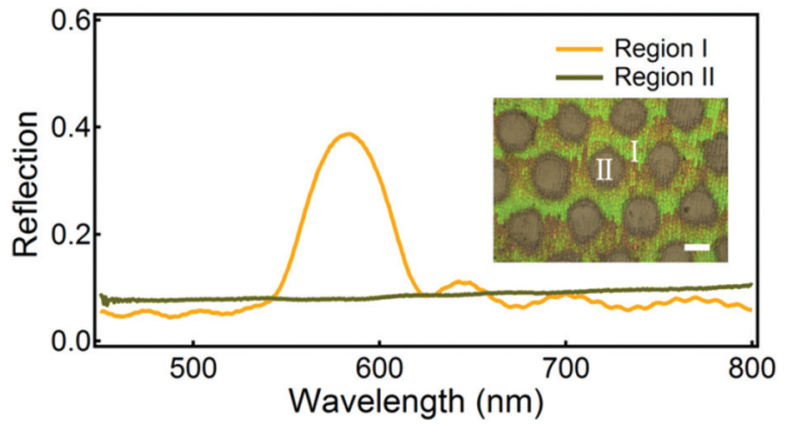

Fig. 2 Reflection spectra of imprinted CPC patterns at $100{ }^{\circ} \mathrm{C}, 30$ bar, $60 \mathrm{~s}$. Inset is the optical image of the imprinted patterns, scale bar is $100 \mu \mathrm{m}$.

upon imprinting. In the imprinted regions, the spheres are deformed into polyhedra ${ }^{40}$ while in the non-imprinted regions their spherical shape is maintained (see Fig. 1c and d). The average distance between the neighboring layers is $203 \mathrm{~nm}$ in the imprinted regions (Fig. 1d) which is $10 \mathrm{~nm}$ less than the non-imprinted location (213 nm) as shown in Fig. 2c. Because of the decreasing lattice distance perpendicular to the surface, a blue-shift of the reflection peak is observed in the imprinted 
regions from $565 \mathrm{~nm}$ (dashed) to $535 \mathrm{~nm}$ (green solid line). In the non-imprinted region, the Poly(St-MMA-AA) nanospheres are also subject to lateral squeezing and partial melting from proximity to the imprinted regions, however the reflection peak produced now slight red-shifts from 565 to $575 \mathrm{~nm}$ (orange solid line).

The colour patterns generated by microimprinting can further be tuned by adjusting the imprinting conditions. The most critical factor is the imprinting temperature. If higher temperatures $\left(100{ }^{\circ} \mathrm{C}\right)$ are applied during imprinting, melting occurs in the imprinted region, thus destroying any photonic structure and colour after peeling off the stamp which leaves only a single colour pattern (inset Fig. 2). The reflection spectra (Fig. 2) show that the reflection peak disappears in the printed region (Region II) while the non-imprinted region (Region I) maintains its light green structural colour with reflection peak at $580 \mathrm{~nm}$. Further increasing the imprinting temperature $\left(110{ }^{\circ} \mathrm{C}\right)$ leads to complete loss of structural colour over the whole film as shown in ESI Fig. S2. $\dagger$

A key factor to successful dual patterning is the pre-annealing step of the CPCs before imprinting, which enhances the mechanical stability of the Poly(St-MMA-AA) CPC on the substrate. Otherwise the weak contact between the hydrophobic Poly(St-MMA-AA) CPC films and the hydrophilic glass substrate causes the patterned regions to easily peel off, resulting in the imprinted regions being lifted off the substrate together with the stamp, as schematically shown in Fig. 3a. The resulting CPCs left behind on the substrate, together with the inverted patterns sticking to the stamp, are shown in the insets of Fig. 3b. The reflection spectra of these corresponding patterns (peaked at 540 and $574 \mathrm{~nm}$ ) are almost fully consistent with those in Fig. 1e.

Based on this type of patterning, we are able to colloidally assemble another layer of any different type of CPC around

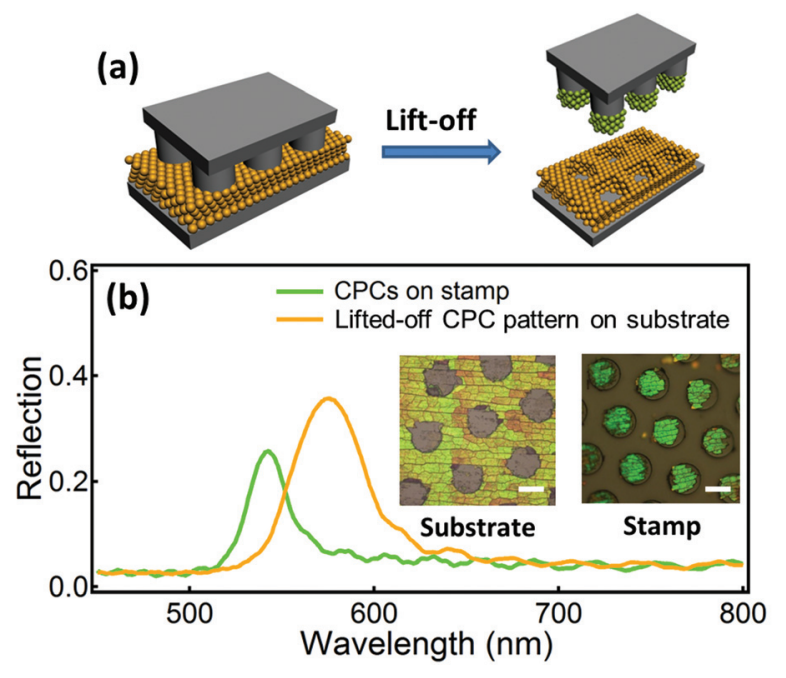

Fig. 3 (a) Scheme of fabrication of CPC patterns by imprint lithography. (b) Reflection spectra of CPC patterns made by soft lithography. Insets are the optical images of CPC patterns on the substrate (left) and on the stamp (right). Scale bars are $100 \mu \mathrm{m}$.
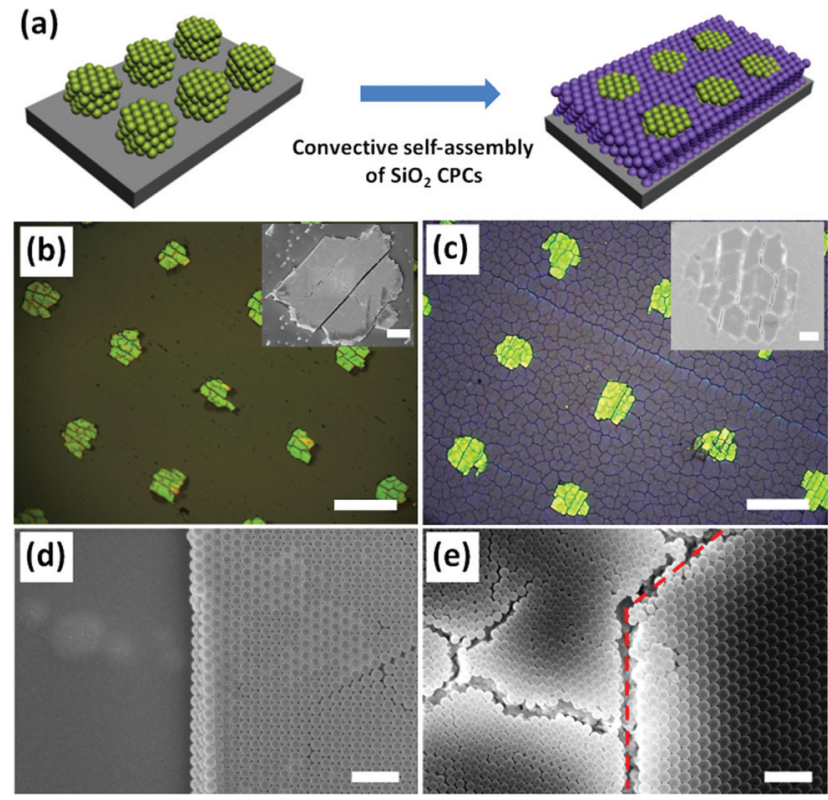

Fig. 4 (a) Scheme to fabricate composite dual CPC patterns made of Poly(St-MMA-AA) and $\mathrm{SiO}_{2}$. (b) Optical image of Poly(St-MMA-AA) CPC patterns and (c) dual patterns of Poly(St-MMA-AA) and $\mathrm{SiO}_{2}$. Scale bars are $100 \mu \mathrm{m}$. Insets are SEM images of the micropatterns, scale bars are $10 \mu \mathrm{m}$. (d, e) Magnified view at the boundary of the CPC patterns in (b, c), red dashed lines in (e) show boundary between the two different sized CPCs, scale bars are $1 \mu \mathrm{m}$.

these lifted-off CPC pads, for example one which is instead made of silica nanospheres. The whole process is depicted in Fig. 4a. The first CPC patterns are formed with a stamp made of circular holes arranged on a square lattice (ESI, Fig. S1b †). After this, the $\mathrm{SiO}_{2}$ CPCs are deposited on the substrate via the convective self-assembly method at low concentration $(0.1 \mathrm{v} / \mathrm{v} \%)$ thereby generating dual patterns formed of $\mathrm{SiO}_{2}$ and Poly(St-MMA-AA) spheres (Fig. 4c). The SEM images in Fig. 4b-e show the details of the micropatterns formed after these two steps. The optical images of Fig. 4(b and c) demonstrate that the silica CPC patterns (blue structural colour) are well infiltrated between the patterns of Poly(St-MMA-AA) CPCs (green structural colour). The SEM images of Fig. 4(d and e) clearly show that the empty space between Poly(St-MMA-AA) patterns is filled with silica CPCs after the deposition step. At the boundary between these two regions, clearly-resolved sphere sizes of 190 and $241 \mathrm{~nm}$ can be identified (Fig. 4e). The reflection spectra of the entire patterned films (ESI Fig. S3 $\dagger$ ) have two reflection peaks located at 419 and $564 \mathrm{~nm}$, representing the photonic bandgaps of Poly(St-MMA-AA) and $\mathrm{SiO}_{2}$ CPCs, respectively.

One unique advantage of the dual patterns made of different materials is that they have different hydrophobicities. This can result in different filling fractions when filled with aqueous liquid, thus resulting in different changes in reflection intensity and peak wavelength. Here water and ethanol are used as two contrasting liquid fillers, which produce distinctive changes in the reflection spectra of patterns as sum- 

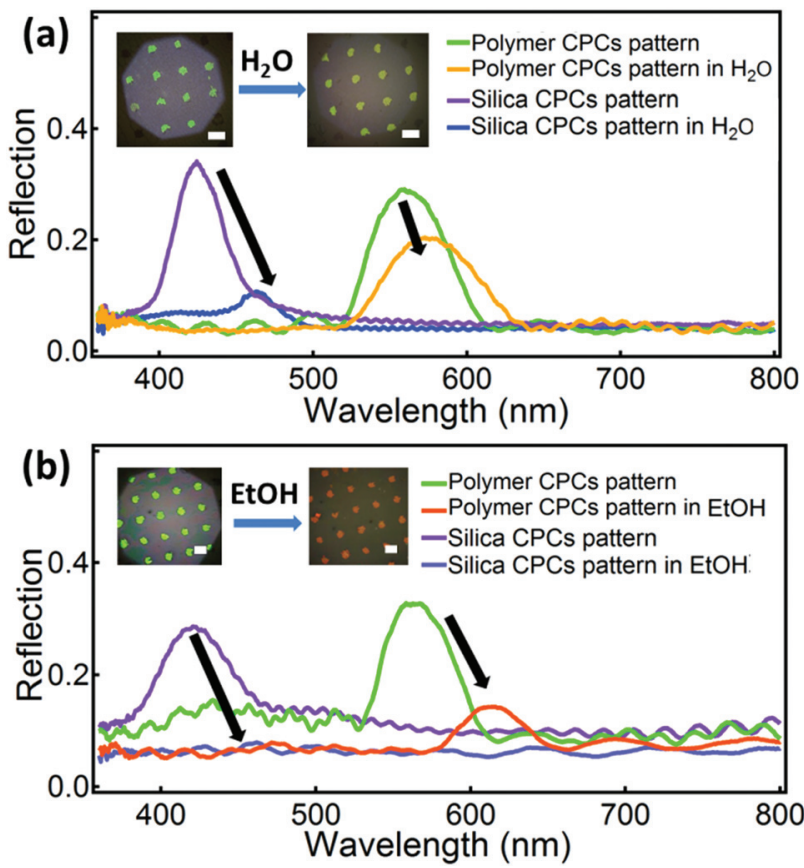

Fig. 5 The reflection spectra of dual CPC patterns made of Poly(StMMA-AA) and $\mathrm{SiO}_{2}$ in response to different liquids, (a) $\mathrm{H}_{2} \mathrm{O}$ and (b) ethanol. Insets are optical images of the CPC patterns before and after liquid infiltration. Scale bars are $100 \mu \mathrm{m}$.

marized in Fig. 5. We selectively detect the spatially-localized reflection spectra of the patterned regions of $\mathrm{SiO}_{2}$ and Poly(St-MMA-AA) by using confocal microscopy, yielding reflection peaks at $423 \mathrm{~nm}$ for $\mathrm{SiO}_{2}$ and $562 \mathrm{~nm}$ for Poly(St-MMA-AA). After water infiltration, the colour of the silica patterns almost fades away completely, while the colour of the Poly(St-MMA-AA) patterns change from green to yellow (optical images in insets of Fig. 5a). The reflection peaks of $\mathrm{SiO}_{2} \mathrm{CPCs}$ red-shift to $462 \mathrm{~nm}$ and the peak intensity decreases to $3.2 \%$ because the infiltration with water lowers the refractive index contrast and increases the average reflective index of the films. In contrast for the Poly(St-MMA-AA) CPC patterns, the reflection peak only red-shifts to $575 \mathrm{~nm}$ while the intensity remains above $15 \%$.

This much reduced shift compared to the silica CPC patterns is mainly attributed to different filling ratios of water into the CPCs, caused by the different surface tension of hydrophilic silica and hydrophobic Poly(St-MMA-AA). The surface of Poly(St-MMA-AA) should be hydrophilic initially due to the presence of poly(acrylate acid), but imprinting above $T_{\mathrm{g}}$ can result in rearrangement of the polymer chains, which makes the surface hydrophobic. For the hydrophilic silica, the water wets well within the interstices of the CPCs, producing more infiltration and lower refractive index contrast. On the other hand, for the hydrophobic Poly(St-MMA-AA) CPCs, the wetting of water within the interstices is poor due to the high surface tension, which results in lower filling ratios and less shift of the reflection peak. If instead ethanol (EtOH) is used as the infiltration solvent, the reflection peaks dramatically
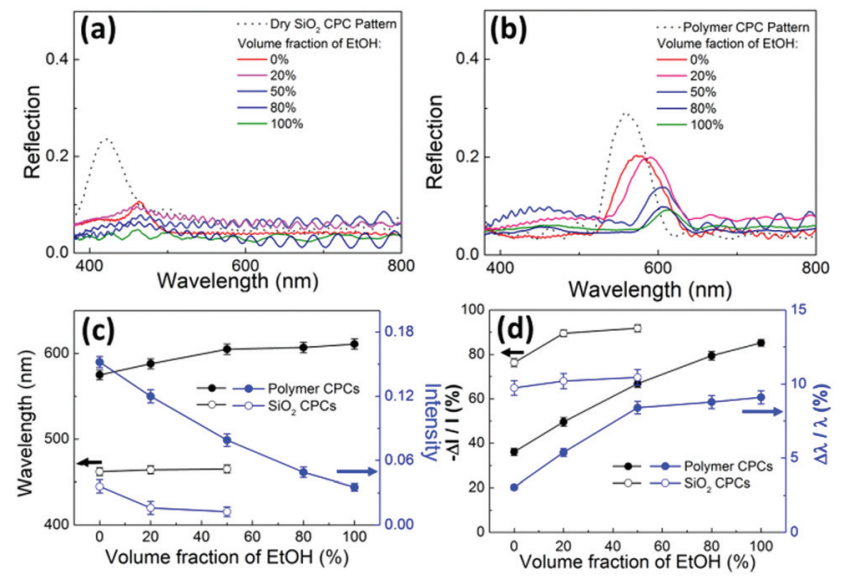

Fig. 6 Reflection spectra of CPC patterns in response to liquid mixtures with different ratios of ethanol and water. (a) $\mathrm{SiO}_{2} \mathrm{CPCs}$, (b) Poly(StMMA-AA) CPCs. (c) Change of wavelength and intensity of the reflection peaks (background subtracted) of the $\mathrm{SiO}_{2}$ and Poly(St-MMA-AA) CPC patterns. (d) Relative intensity and shift of reflection peaks for different ratios of solvents.

red-shift from 563 to $614 \mathrm{~nm}$ and the colour of the Poly(StMMA-AA) CPC patterns changes from green to red (inset Fig. 5b). The reflection peak of silica CPC patterns now almost vanishes (Fig. 5b), due to the close match of refractive indices between EtOH and silica. Such shifts of peak wavelength and changes of intensity are fully reversible and can be repeated many times as shown in ESI Fig. S4. $\dagger$

By changing the volume ratios of mixtures of $\mathrm{H}_{2} \mathrm{O}$ and $\mathrm{EtOH}$, the surface tension of the liquid can be tuned, which changes their infiltration ratios within the CPCs. Hence the reflection peaks of both Poly(St-MMA-AA) and $\mathrm{SiO}_{2} \mathrm{CPC}$ patterns now red-shift gradually with increasing volume ratio of $\mathrm{EtOH}$ (Fig. 6a and b). Note, it is harder to identify the reflection peaks from $\mathrm{SiO}_{2} \mathrm{CPC}$ patterns above $50 \% \mathrm{v} / \mathrm{v}$. The change of reflection peak positions and intensity are summarized in Fig. 6c. Noteworthy is that these two patterns show nonuniform shifts of the reflection peaks, from 575 to $611 \mathrm{~nm}$ (in Poly(St-MMA-AA) CPC patterns) and 462 to $465 \mathrm{~nm}$ (in $\mathrm{SiO}_{2}$ CPC patterns, before disappearing), when varying the solvent.

These red-shifts have a contribution from the small increase in the average refractive index expected when replacing water with EtOH. However the peak position for Poly(StMMA-AA) CPCs is predicted to be $600 \mathrm{~nm}$ after full infiltration of water, which is much larger than the experimental value obtained here (shown in ESI Table S1 $\dagger$ ). Therefore, rather than the increase of overall refractive index of the liquid being important for the red-shift, it is instead the improved filling caused by the lower surface tension which is produced as the content of EtOH is increased. It is apparent in Fig. 6d that the kink in the reflection peak tuning of Poly(St-MMA-AA) CPCs (at volume fraction $50 \%$ ) is at the expected point when the CPCs are fully infiltrated by the solvents (Fig. 7c). The slower rate of red-shift observed after this point should then be caused solely by the increase of the average refractive index. The wetting 

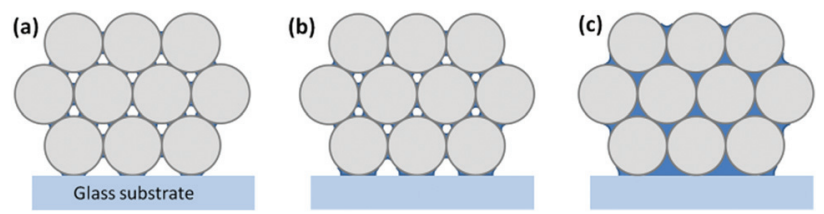

Fig. 7 Infiltration profiles of the solvent mixtures within Poly(StMMA-AA) CPCs. The volume fractions of EtOH are (a) $0 \%$ (b) $20 \%$, and (c) $50 \%$.

profiles of the solvents with different volume fractions of EtOH are schematically shown in Fig. 7 to clarify this coupling to the photonic tuning. Additional evidence for the infiltration mechanism is that the intensity and peak position of Poly(St-MMA-AA) CPC patterns depend strongly on solvent composition while little change is seen for $\mathrm{SiO}_{2} \mathrm{CPC}$ patterns (Fig. 6d). This indicates that changes of refractive index mostly arise from solvent loading effects. Changes of surface tension inducing changes of filling ratio play the dominant role in colour changes of this dual CPC pattern system.

The size feature of the patterns could be enlarged for a number of potential applications. One is based on a counterfeit detection mechanism in which a film dipped into different alcohols changes colour selectively to reveal different symbols or text. This can be utilized in two ways. It can be used to verify the specific gravity or solvent concentration of commercial alcohols in a very quick and readily discernible measurement. It can also be simply used to verify a polymer-based currency banknote or credit card, by immersion in water and vodka, and checking the different symbols that appear. A wide variety of optical effects can be developed from this patterning protocol to extend the range of operations.

\section{Experimental}

\section{Microimprint lithography of CPCs made of Poly(St-MMA-AA)}

The Poly(St-MMA-AA) latex spheres of diameter $240 \mathrm{~nm}$ were synthesized as in previous reports ${ }^{41}$ and self-assembled to form 3D CPCs via convective self-assembly. ${ }^{42}$ Typically $0.1 \mathrm{wt} \%$ of Poly(St-MMA-AA) beads were dispersed in water, and a vertically-oriented glass slide inserted and left to dry under constant temperature of $65{ }^{\circ} \mathrm{C}$ for $24 \mathrm{~h}$. The CPCs of Poly(StMMA-AA) are coated onto the surface of the glass slides during this drying process. Afterwards, the Poly(St-MMA-AA) CPCs can be pre-annealed at $90{ }^{\circ} \mathrm{C}$ for $30 \mathrm{~min}$.

The imprinting condition used mostly here is $90^{\circ} \mathrm{C}, 20$ bar for $30 \mathrm{~s}$ and is implemented and monitored with an Obducat Nanoimprinter.

\section{Fabrication of composite CPC patterns}

For the composite patterns, the Poly(St-MMA-AA) CPC patterns were first created by imprint lithography of the non-annealed samples of Poly(St-MMA-AA) CPCs. The substrate on which the Poly(St-MMA-AA) CPC patterns were laid down was immersed in an ethanol dispersion of silica beads (of diameter $185 \mathrm{~nm}$, at $0.1 \mathrm{wt} \%$ ) and placed in an oven preheated to $36^{\circ} \mathrm{C}$. After $24 \mathrm{~h}$ the dispersion was dried and the silica beads selfassembled around the Poly(St-MMA-AA) CPCs patterns, to form dual composite CPC patterns.

\section{Characterisations}

The samples were characterized using an optical microscope (BX51 Olympus) coupled with an optical fiber-coupled spectrometer (QE65000 Ocean Optics) to provide reflection spectra recorded at many spatial positions across the patterns. The micromorphology of the CPC patterns was examined with a Zeiss SEM (LEO 1530VP) at an accelerating voltage of $5 \mathrm{kV}$.

A mixture of ethanol and water with different ratios was applied to examine the different sensitivity of the composite dual patterns, which is related to the relative intensity and shift of the reflection peaks. The \% change of the reflection intensity is determined by from the reflection maximum before/after liquid infiltration. The fractional change of the reflection wavelength $\Delta \lambda / \lambda$ is determined from the peak spectral shift.

The expected reflection peak position is estimated by using the formula ${ }^{43}$

$$
m \lambda=2 d_{111} \sqrt{f n_{\text {sphere }^{2}+(1-f) n_{\text {fill }}^{2}}}
$$

where $m$ is the order of Bragg diffraction, $\lambda$ is the wavelength of the stop band maximum, $d_{111}$ is the interlayer spacing of (111) crystalline planes, which is estimated as $(2 / 3)^{1 / 2} D$, where $D$ is the diameter of the spheres, and $f$ is the volume fraction occupied by colloids, which is approximately 0.74 for close-packed spheres. Here $n_{\text {sphere }}$ is the refractive index of the colloids, and $n_{\text {fill }}$ is the average refractive index of the solvent which can be roughly estimated by

$$
n_{\text {fill }}=\sqrt{n_{\mathrm{EtOH}^{2}} \varphi+n_{\mathrm{H}_{2} \mathrm{O}^{2}}(1-\varphi)}
$$

where $\varphi$ is the volume fraction of EtOH in the mixture, and $n_{\mathrm{EtOH}}$ and $n_{\text {water }}$ are the refractive indices of ethanol and water respectively.

The reflection intensity is estimated with formula

$$
I \propto \frac{\Delta n}{n_{\text {eff }}}
$$

where $\Delta n$ is the difference of refractive index between spheres and the infiltrating medium, $n_{\text {eff }}$ is the effective refractive index of the entire CPC film.

\section{Conclusions}

In this article, we introduced a facile way for large scale patterning of CPCs via microimprint lithography. Two scenarios were discussed. If the CPCs are pre-annealed, microimprinting leads to compression of the imprinted region thereby changing the lattice separation normal to the surface, which results in dual colour patterns. If the CPCs are not preannealed, microimprinting selectively peels the contacted 
region off the substrate, thus forming negative CPCs patterns on the substrate. The Poly(St-MMA-AA) CPC patterns are shown to facilitate further assembly of other types of CPCs, here made of silica. In this way, we created dual CPCs patterns with different composition and different structural colours. Because the hydrophobicity of these two patterns differs due to the chemical nature of the colloids, the silica CPC patterns were much more easily wetted by water than their Poly(St-MMA-AA) counterparts, which produces different optical response to solvents. Specifically, the red-shift and intensity drop of the reflection peak from Poly(St-MMA-AA) CPC patterns are much smaller than those from $\mathrm{SiO}_{2} \mathrm{CPC}$ patterns because of the lower filling of $\mathrm{H}_{2} \mathrm{O}$ into the hydrophobic interstices of Poly(St-MMA-AA) spheres. With increasing ratios of EtOH in $\mathrm{H}_{2} \mathrm{O}$, the surface tension of the liquid drops, hence the filling ratio increases and the reflection peak gradually red-shifts while its intensity decreases. Instead for silica CPC patterns, the change of liquid composition does not strongly change the filling ratio and only slightly increases the refractive index of the filling liquid, leading to minimal colour changes. Such a complex patterning system with different component sensitivities to solvent can potentially be used for sensing, image display and antifouling materials.

\section{Acknowledgements}

We acknowledge financial support from EPSRC grant EP/ G060649/1，EP/I012060/1，EP/J007552/1，EP/L027151/1， ERC grant LINASS 320503, EMATTER 280078.

\section{Notes and references}

1 A. Arsenault, S. B. Fournier-Bidoz, B. Hatton, H. Miguez, N. Tetrault, E. Vekris, S. Wong, S. M. Yang, V. Kitaev and G. A. Ozin, J. Mater. Chem., 2004, 14, 781-794.

2 J. D. Joannopoulos, P. R. Villeneuve and S. H. Fan, Nature, 1997, 386, 143-149.

3 P. V. Braun and P. Wiltzius, Nature, 1999, 402, 603-604.

4 G. Subramania, K. Constant, R. Biswas, M. M. Sigalas and K.-M. Ho, Appl. Phys. Lett., 1999, 74, 3933-3935.

5 J. E. G. J. Wijnhoven and W. L. Vos, Science, 1998, 281, 802804.

6 S.-H. Kim, S. Y. Lee, S.-M. Yang and G.-R. Yi, NPG Asia Mater., 2011, 3, 25-33.

7 A. T. Skjeltorp and P. Meakin, Nature, 1988, 335, 424-426.

8 E. Pavarini, L. C. Andreani, C. Soci, M. Galli, F. Marabelli and D. Comoretto, Phys. Rev. B: Condens. Matter, 2005, 72, 045102.

9 T. Ding, Y. Long, K. Zhong, K. Song, G. Yang and C.-H. Tung, J. Mater. Chem. C, 2014, 2, 4100-4111.

10 H. Hu, J. Tang, H. Zhong, Z. Xi, C. Chen and Q. Chen, Sci. Rep., 2013, 3, 1484.

11 H. Kim, J. Ge, J. Kim, S.-E. Choi, H. Lee, H. Lee, W. Park, Y. Yin and S. Kwon, Nat. Photonics, 2009, 3, 534-540.
12 I. B. Burgess, L. Mishchenko, B. D. Hatton, M. Kolle, M. Lončar and J. Aizenberg, J. Am. Chem. Soc., 2011, 133, 12430-12432.

13 L. Wang, J. Wang, Y. Huang, M. Liu, M. Kuang, Y. Li, L. Jiang and Y. Song, J. Mater. Chem., 2012, 22, 2140521411.

14 H. S. Lee, T. S. Shim, H. Hwang, S.-M. Yang and S.-H. Kim, Chem. Mater., 2013, 25, 2684-2690.

15 D. Yang, S. Ye and J. Ge, Adv. Funct. Mater., 2014, 24, 31973205.

16 S. Y. Lee, S.-H. Kim, H. Hwang, J. Y. Sim and S.-M. Yang, Adv. Mater., 2014, 26, 2391-2397.

17 J. H. Moon, A. Small, G.-R. Yi, S.-K. Lee, W.-S. Chang, D. J. Pine and S.-M. Yang, Synth. Met., 2005, 148, 99-102.

18 S. K. Lee, G. R. Yi, J. H. Moon, S. M. Yang and D. J. Pine, Adv. Mater., 2006, 18, 2111-2116.

19 Z.-Z. Gu, A. Fujishima and O. Sato, Angew. Chem., Int. Ed., 2002, 114, 2171-2174.

20 C. A. Fustin, G. Glasser, H. W. Spiess and U. Jonas, Adv. Mater., 2003, 15, 1025-1028.

21 C.-A. Fustin, G. Glasser, H. W. Spiess and U. Jonas, Langmuir, 2004, 20, 9114-9123.

22 A. van Blaaderen, R. Ruel and P. Wiltzius, Nature, 1997, 385, 321-324.

23 J. Ye, R. Zentel, S. Arpiainen, J. Ahopelto, F. Jonsson, S. G. Romanov and C. M. Sotomayor Torres, Langmuir, 2006, 22, 7378-7383.

24 T. Ding, L. Luo, H. Wang, L. Chen, K. Liang, K. Clays, K. Song, G. Yang and C.-H. Tung, J. Mater. Chem., 2011, 21, 11330-11334.

25 S. M. Yang and G. A. Ozin, Chem. Commun., 2000, 25072508.

26 B. Varghese, F. C. Cheong, S. Sindhu, T. Yu, C.-T. Lim, S. Valiyaveettil and C.-H. Sow, Langmuir, 2006, 22, 82488252.

27 S. Arpiainen, F. Jonsson, J. R. Dekker, G. Kocher, W. Khunsin, C. M. S. Torres and J. Ahopelto, Adv. Funct. Mater., 2009, 19, 1247-1253.

28 A. M. Brozell, M. A. Muha and A. N. Parikh, Langmuir, 2005, 21, 11588-11591.

29 H.-L. Li and F. Marlow, Chem. Mater., 2005, 17, 3809-3811.

30 J. Park, J. Moon, H. Shin, D. Wang and M. Park, J. Colloid Interface Sci., 2006, 298, 713-719.

31 L. Cui, Y. Li, J. Wang, E. Tian, X. Zhang, Y. Zhang, Y. Song and L. Jiang, J. Mater. Chem., 2009, 19, 5499-5502.

32 J. Wang, L. Wang, Y. Song and L. Jiang, J. Mater. Chem. C, 2013, 1, 6048-6058.

33 K. Burkert, T. Neumann, J. Wang, U. Jonas, W. Knoll and H. Ottleben, Langmuir, 2007, 23, 3478-3484.

34 (a) T. Ding, Q. Zhao, S. K. Smoukov and J. J. Baumberg, Adv. Opt. Mater., 2014, 2, 1098-1104; (b) T. Ding, L. Chen, Y. Long and K. Song, RSC Advances, 2014, DOI: 10.1039/ C4RA12958J.

35 X. Yan, J. Yao, G. Lu, X. Chen, K. Zhang and B. Yang, J. Am. Chem. Soc., 2004, 126, 10510-10511. 
36 J. Yao, X. Yan, G. Lu, K. Zhang, X. Chen, L. Jiang and B. Yang, Adv. Mater., 2004, 16, 81-84.

37 B. Hatton, L. Mishchenko, S. Davis, K. H. Sandhage and J. Aizenberg, Proc. Natl. Acad. Sci. U. S. A., 2010, 107, 10354-10359.

38 J. Zhou, J. Wang, Y. Huang, G. Liu, L. Wang, S. Chen, X. Li, D. Wang, Y. Song and L. Jiang, NPG Asia Mater., 2012, 4, e21.

39 Y. Huang, J. Zhou, B. Su, L. Shi, J. Wang, S. Chen, L. Wang, J. Zi, Y. Song and L. Jiang, J. Am. Chem. Soc., 2012, 134, 17053-17058.
40 Z. Q. Sun, X. Chen, J. H. Zhang, Z. M. Chen, K. Zhang, X. Yan, Y. F. Wang, W. Z. Yu and B. Yang, Langmuir, 2005, 21, 8987-8991.

41 J. Wang, J. Hu, Y. Wen, Y. Song and L. Jiang, Chem. Mater., 2006, 18, 4984-4986.

42 (a) P. Jiang, J. F. Bertone, K. S. Hwang and V. L. Colvin, Chem. Mater., 1999, 11, 2132-2140; (b) N. D. Denkov, O. D. Velev, P. A. Kralchevsky, I. B. Ivanov, H. Yoshimura and K. Nagayama, Langmuir, 1992, 8, 3183-3190.

43 G. Subramania, R. Biswas, K. Constant, M. M. Sigalas and K. M. Ho, Phys. Rev. B: Condens. Matter, 2001, 63, 235111. 